\title{
CARACTERIZAÇÃO DO USO DE MEDICAMENTOS ASSOCIADOS PARA HIPERTENSÃO E/OU DIABETES NO MUNICÍPIO DE CATUNDA, CEARÁ
}

CHARACTERIZATION OF THE USE OF ASSOCIATED DRUGS FOR HYPERTENSION AND/OR DIABETES IN THE MUNICIPALITY OF CATUNDA, CEARÁ

CARACTERIZACIÓN DEL USO DE MEDICINAS ASOCIADAS PARA HIPERTENSIÓN Y/O DIABETES EN EL MUNICIPIO DE CATUNDA, CEARÁ

\section{Como Citar:}

Costa MMB, Linhares MI.

Caracterização do uso de medicamentos associados para hipertensão e/ou diabetes no município de Catunda, Ceará. Sanare (Sobral, Online). 2021; 20(2):47-53.

Descritores:

Hipertensão; Diabetes; Saúde pública.

Descriptors: Hypertension; Diabetes; Public health.

Descriptores: Hipertensión; Diabetes; Salud pública.

Submetido: $16 / 10 / 2021$

Aprovado: 24/11/2021

Autor(a) para Correspondência: Maria Morgana Bezerra Costa Centro Universitário INTA-UNINTA Rua Antônio Calisto dos Santos, 622, Centro, Catunda, Ceará CEP: 62297-000 E-mail: morgana_bcosta@hotmail.com

\section{RESUMO}

A hipertensão e o diabetes, doenças crônicas não transmissiveis, com fácil diagnóstico e um arsenal terapêutico à disposição, têm repercutido consideravelmente nos indicadores de morbimortalidade. Assim, o estudo objetiva caracterizar o uso de medicamentos associados para hipertensão $e$ diabetes no município de Catunda, Ceará. Pesquisa retrospectiva, documental, com abordagem quantitativa, realizada em uma Unidade Básica de Saúde localizada em Catunda, Ceará, em que foram analisados 137 registros de prescrição de tratamento para hipertensão e/ou diabetes, no período de outubro de 2018 a abril de 2020. Observou-se que as prescrições se apresentavam (100\%) de forma legível e (97,8\%) com dados completos, o profissional prescritor era o médico clínico geral (89,8\%), com atendimento vinculado ao setor público. 0 perfil de pacientes foi prevalente para mulheres (72,3\%). Sobre os fármacos, verificou-se a prevalência (37\%) do uso de hidroclorotiazida (anti-hipertensivo) e (56\%) de metformina (hipoglicemiante). Em relação às interações medicamentosas, a mais frequente foi enalapril + glibenclamida, seguida de hidroclorotiazida + glibenclamida e propranolol + metformina. Esses achados constituem informações importantes que podem contribuir com o planejamento e execução de políticas públicas de saúde, assim como para a redução de outras complicações associadas, como doenças cardiovasculares.

1. Farmacêutica. Pós-graduanda em Farmácia Clínica e Prescrição Farmacêutica. Centro Universitário INTA UNINTA. E-mail: morgana_bcosta@hotmail.com ORCID: https://orcid.org/0000-0002-2517-0474

2. Farmacêutica. Doutora em Farmacologia pela Universidade Federal do Ceará. Centro Universitário INTA - UNINTA. E-mail: misabel.linhares@gmail.com 0RCID: https://orcid.org/0000-0003-2337-9810

Cert. de Redação Científica: Central das Revisões. Edição de texto: Karina Maria M. Machado. Revisão de provas: Texto definitivo validado pelos(as) autores (as). 


\section{ABSTRACT}

Hypertension and diabetes, non-communicable chronic diseases, with easy diagnosis and a therapeutic arsenal available, have had considerable repercussions on morbimortality indicators. Thus, the study aims to characterize the use of associated drugs for hypertension and diabetes in the city of Catunda, Ceará. Retrospective, documentary research, with a quantitative approach, carried out in a Basic Health Unit located in Catunda, Ceará, in which 137 records of treatment prescriptions for hypertension and/or diabetes were analyzed, in the period from 0 ctober 2018 to April 2020. We observed that of the prescriptions (100\%) were legible, and (97.8\%) had complete data, and the prescribing professional was a general practitioner (89.8\%) working in the public sector. The prevalent patient profile was female (72.3\%). Regarding drugs, there was a prevalent use (37\%) of hydrochlorothiazide (anti-hypertensive agent), and (56\%) of metformin (hypoglycemic agent). Regarding drug interactions, the most frequent was enalapril + glibenclamide, followed by hydrochlorothiazide + glibenclamide and propranolol + metformin. These findings constitute important information that can contribute to the planning and execution of public health policies, and to the reduction of other related complications, such as cardiovascular diseases.

\section{RESUMEN}

La hipertensión y la diabetes, enfermedades crónicas no transmisibles, con fácil diagnóstico y un arsenal terapéutico a la disposición, tiene repercutido considerablemente en los indicadores de morbimortalidad. Así, el estudio tiene como objetivo caracterizar el uso de medicinas asociadas para la hipertensión y diabetes en el municipio de Catunda, Ceará. Investigación retrospectiva, documental, con abordaje cuantitativo, realizada en una Unidad Básica de Salud ubicada en Catunda, Ceará, en la cual fueron analizados 137 registros de prescripción de tratamiento para hipertensión y/o diabetes, en el periodo de octubre de 2018 hasta abril de 2020. Se observó que las prescripciones se presentaban (100\%) de forma legible y $(97,8 \%)$ con datos completos, el profesional prescriptor era el medico clínico general $(89,8 \%)$, con atendimiento vehiculado al sector público. El perfil de pacientes fue prevalente para mujeres (72,3\%). Sobre los fármacos, se verificó la prevalencia (37\%) del uso de hidroclorotiazida (antihipertensivo) y (56\%) de metformina (hipoglicemiante). En relación a las interacciones medicamentosas, la más frecuente fue enalapril + glibenclamida y propanolol + metformina. Lo que ha sido encontrado constituye informaciones importantes que pueden contribuir con el plan y ejecución de políticas públicas de salud, así como para la reducción de otras complicaciones asociadas, como enfermedades cardiovasculares.

\section{INTRODUÇÃ O}

0 Brasil tem passado por um processo de mudança epidemiológica em que as doenças crônicas não transmissiveis (DCNT) têm sido visualizadas como o principal motivo de morbimortalidade, além de acarretarem forte impacto econômico. Dentre essas condições clínicas crônicas, a hipertensão arterial sistêmica (HAS) e o diabetes mellitus (DM) têm se destacado pelo seu considerável potencial de risco associado para doença renal, acidente vascular encefálico e outras doenças cardiovasculares ${ }^{1}$.

A HAS é uma situação clínica que envolve diversos fatores e se caracteriza por uma elevação dos níveis pressóricos, sendo capaz de ultrapassar os $140 / 90 \mathrm{mmHg}$, de modo que o coração precisa fazer um maior esforço para possibilitar a distribuição do sangue pelo corpo. A HAS pode ser intensificada pelo surgimento de alguns fatores de risco (FR), tais como: diabetes mellitus, obesidade abdominal, dislipidemia e intolerância à glicose ${ }^{3}$. A DM é uma doença definida por insuficiência ou dificuldade de absorção na produção de insulina, que é responsável por regular a glicose no sangue fornecendo energia para o corpo, sendo classificada como DM tipo 1 e tipo 2. Ela ocupa $07^{\circ}$ lugar nas principais causas de óbito no mundo. Estudos relatam que a predominância de pessoas com hipertensão associada à diabetes é próxima do dobro quando comparada aos não diabéticos, havendo risco maior de doença cardiovascular em pacientes que são acometidos por ambas as patologias ${ }^{2,4}$.

Em relação aos medicamentos usados no tratamento da HAS, podem ser utilizadas diferentes classes, tais como: diuréticos, inibidores adrenérgicos, betabloqueadores, inibidores da enzima conversora da angiotensina, vasodilatadores diretos, bloqueadores do canal de cálcio e inibidores diretos da renina. Na DM, são usados, principalmente, as biguanidas e os derivados da ureia. 0 tratamento está relacionado tanto à manutenção contínua pelos pacientes através do uso de medicamentos 
prescritos quanto à mudança do estilo de vida (MEV), a partir da realização, de forma consciente, das ações orientadas quanto aos hábitos de vida, como atividade física e dieta ${ }^{5}$.

A baixa aceitação ao tratamento com o uso dos medicamentos para HAS e DM é um dos principais motivos da diminuição do benefício clínico e manejo das doenças, ocasionando possíveis problemas de saúde, dificuldades psicossociais e redução da qualidade de vida. Sendo assim, tais complicações resultam no agravamento do quadro, levando a uma busca de serviços em saúde de emergência e hospitalizações ${ }^{6}$.

Este estudo propõe-se a caracterizar o uso de medicamentos associados para HAS e DM no município de Catunda-CE, desenvolvendo estratégias educativas com o auxílio dos gestores de saúde do município. Com isso, melhorar a qualidade de vida da população, as implicações sociais e, além de contribuir para a planificação da saúde do município, melhorar o conhecimento da população quanto aos riscos relacionados à $H A S$ e DM, na tentativa de retardar ou prevenir complicações decorrentes da associação dessas patologias. Diante do exposto, o objetivo deste artigo foi: caracterizar o uso de medicamentos associados para hipertensão arterial e diabetes no município de Catunda, Ceará.

\section{METODOLOGIA}

Trata-se de um estudo retrospectivo, descritivo, documental e quantitativo. A população do estudo consistiu em 137 registros de prescrição que continham o uso associado de medicamentos para HAS e DM de pacientes de uma Unidade Básica de Saúde (UBS) localizada no município de Catunda, Ceará, a $256 \mathrm{~km}$ da capital do estado, Fortaleza.

As variáveis que foram avaliadas a partir das prescrições retidas na UBS foram: os sexos dos pacientes que recebem medicamentos para HAS e DM; a caracterização dos medicamentos utilizados quanto à classe farmacológica; os aspectos das prescrições; especialidade do prescritor; origem do atendimento; quantidade de pacientes com associação de HAS e DM; e as possíveis interações medicamentosas. Ao final do periodo de coleta, existiam 137 prescrições retidas na UBS, referentes aos meses de outubro de 2018 a abril de 2020 .

Foram incluídos no estudo os indivíduos com idade superior ou igual a 18 anos e que fazem acompanhamento na UBS, e os que possuem associação, concomitante, de HAS e DM. Foram excluídos do estudo os indivíduos com idade inferior a 18 anos, os que não possuem associação de HAS e DM e os que fazem uso de insulina.

Para melhor análise e compreensão dos resultados obtidos, utilizou-se o programa Microsoft Office Excel ${ }^{\circledR}$, em que os dados foram organizados em tabelas e quadros de acordo com as informações obtidas a partir dos receituários.

A pesquisa foi realizada de acordo com a Resolução do Conselho Nacional de Saúde n. ${ }^{0}$ 466/2012, de 12 de dezembro de 2012, e suas Diretrizes e Normas que regulamentam a pesquisa envolvendo seres humanos no Brasil?. 0 projeto foi cadastrado na Plataforma Brasil para o parecer do Comitê de Ética em Pesquisa, e obteve parecer aprovado com o número 4.254 .552 e CAAE 33716820.7.0000.8109.

\section{RESULTADOS}

0 s dados apresentados são referentes às prescrições de medicamentos associados para HAS e DM retidas na farmácia da Unidade Básica de Saúde em Catunda, Ceará, distribuídos de acordo com sexo, aspectos da prescrição, quanto à especialidade do prescritor, à origem do atendimento, à classe farmacológica e possíveis interações medicamentosas.

Durante o período de coleta de dados foram analisadas 137 prescrições de tratamento para HAS e/ou DM, verificando a coexistência ou não de associação de fármacos, sendo 99 prescrições direcionadas ao sexo feminino $(72,3 \%)$ e 38 a indivíduos do sexo masculino $(27,7 \%)$. No que se refere aos aspectos das prescrições, observou-se que todas elas apresentavam-se legiveis (100\%). Em relação ao preenchimento das prescrições, 134 estavam completas $(97,8 \%)$ e apenas três prescrições estavam incompletas $(2,2 \%)$, por falta de algum dado presente na prescrição, por exemplo, porfalta da data. Quanto à especialidade do prescritor, identificou-se o predomínio de 123 (89,8\%) prescrições realizadas por clínico geral, e 14 foram prescritas por outras especialidades $(10,2 \%)$, entre elas, cardiologistas e endocrinologistas. A análise quanto à origem do atendimento descreve que 131 prescrições foram de origem do setor público $(95,6 \%)$ e apenas seis do setor privado $(4,4 \%)$. A análise desses dados possibilitou a descrição de algumas características, resumidas na Tabela 1. 
Tabela 1. Caracterização das prescrições retidas na UBS.

\begin{tabular}{lcc}
\hline Variáveis & N $=137$ & $\%$ \\
\hline Sexo & & $72,3 \%$ \\
\hline Feminino & 99 & $27,7 \%$ \\
Masculino & 38 & $100 \%$ \\
Aspecto da prescrição & 137 & $0 \%$ \\
Legível & 0 & $97,8 \%$ \\
Ilegível & 134 & $2,2 \%$ \\
Completa & 3 & $89,8 \%$ \\
Incompleta & & $10,2 \%$ \\
Especialidade do prescritor & 123 & 14 \\
Clínico geral & & $95,6 \%$ \\
Outros & 131 & $4,4 \%$ \\
\hline Origem do atendimento & 6 & \\
\hline Público & & \\
Privado & & \\
\hline
\end{tabular}

Fonte: Dados da pesquisa.

Em relação à classe de medicamentos mais utilizada para a hipertensão, considerando que havia várias prescrições com mais de um anti-hipertensivo prescrito, pode-se observar que os diuréticos tiazídicos (Hidroclorotiazida) foram os mais indicados, com 68 prescrições contendo esse medicamento (37\%); antagonista da angiotensina II (Losartana) em 59 prescrições (32\%); inibidores da enzima conversora de angiotensina (Enalapril) em 23 prescrições (12\%); bloqueadores seletivos dos canais de cálcio (Anlodipino) em nove prescrições ( $5 \%$ ); betabloqueadores seletivos (Atenolol) em oito prescrições (4\%); diuréticos de alça (Furosemida) em sete prescrições ( $4 \%$ ); agentes alfa e beta bloqueadores (Carvedilol) em seis prescrições ( $3 \%$ ); betabloqueadores não seletivos (Propanolol) com quatro prescrições (2\%); agentes poupadores de potássio (Espironolactona) em uma prescrição (1\%), conforme destacado na Tabela 2.

Tabela 2. Distribuição de medicamentos para HAS e classe farmacológica quanto às prescrições.

\begin{tabular}{lccc}
\hline Classe farmacológica & Denominação genérica & F & $\%$ \\
\hline Diuréticos tiazídicos & Hidroclorotiazida & 68 & $37 \%$ \\
\hline Antagonista da angiotensina II & Losartana & 59 & $32 \%$ \\
\hline Inibidores da enzima conversora de angiotensina & Maleato de Enalapril & 123 & $12 \%$ \\
\hline $\begin{array}{l}\text { Bloqueadores seletivos dos canais de cálcio } \\
\text { Derivados da diidropiridina }\end{array}$ & Besilato de Anlodipino & 9 & $5 \%$ \\
Betabloqueadores seletivos & Atenolol & 8 & $4 \%$ \\
Diuréticos (de alça) & Furosemida & 7 & $4 \%$ \\
Agentes alfa e beta bloqueadores & Carvedilol & 6 & $3 \%$ \\
Betabloqueadores não seletivos & Propanolol & 4 & $2 \%$ \\
Agentes poupadores de potássio & Espironolactona & 1 & $1 \%$ \\
\hline
\end{tabular}

Fonte: Dados da pesquisa.

Em relação à classe dos medicamentos para DM, considerando o fato de que havia várias prescrições com mais de um hipoglicemiante prescrito, pode-se observar que as classes mais prescritas foram as biguanidas (Metformina), encontradas em 92 prescrições (56\%); as sulfonamidas, caracterizadas por Glicazida, com 8 prescrições ( $5 \%)$; e por Glibenclamida, com 63 prescrições (39\%), como mostra a Tabela 3. 
Tabela 3. Distribuição de medicamentos para DM e classes farmacológicas quanto às prescrições.

\begin{tabular}{lccc}
\hline Classe farmacológica & Denominação genérica & F & $\%$ \\
\hline Biguanidas & Cloridrato de Metformina & 92 & $56 \%$ \\
\multirow{2}{*}{ Derivados da ureia, sulfonamidas } & Glibenclamida & 63 & $39 \%$ \\
& Gliclazida & 8 & $5 \%$ \\
\hline
\end{tabular}

Fonte: Dados da pesquisa.

Avaliando os medicamentos prescritos nos receituários é possível observar que todos os pacientes utilizam dois medicamentos ou mais, favorecendo as possíveis interações medicamentosas. Dentre as combinações mais encontradas, destacam-se: Enalapril + Glibenclamida, Hidroclorotiazida + Glibenclamida, Propanolol + Metfmormina, Enalapril + Metformina e Hidroclorotiazida + Metformina, sendo essas consideradas interações de relevância clínica significativa, como mostra o Quadro 1.

Quadro 1. Distribuição das possíveis interações entre os medicamentos para HAS e DM a partir das prescrições.

\begin{tabular}{|l|l|}
\hline Interações medicamentosas & Risco potencial \\
\hline Enalapril + Glibenclamida & Hipoglicemia, hiperglicemia ou hipertensão \\
\hline Glibenclamida + Hidroclorotiazida & Redução do efeito hipoglicemiante \\
\hline Propanolol + Metformina & Hipoglicemia, hiperglicemia ou hipertensão \\
\hline Enalapril + Metformina & Acidose láctica hipercalêmica \\
\hline Metformina + Hidroclorotiazida & Hiperglicemia e intolerância à glicose \\
\hline
\end{tabular}

Fonte: Adaptado de Amaral e Perassolo ${ }^{8}$.

\section{DISCUSSÃO}

Corroborando o achado, um estudo que buscou avaliar o uso de medicamentos para hipertensão e diabetes, em todas as regiões do Brasil, observou que a prevalência do uso de fármacos por mulheres foi de $28,1 \%$, sendo esse número de maior relevância quando comparado com os homens $(18,5 \%)^{9}$.

No presente estudo, houve uma quantidade pequena de ilegibilidade e isso está relacionado ao fato de que na UBS os prescritores já liberam as receitas digitalizadas; desse modo, não há dificuldade de identificação dos medicamentos, sendo importante enfatizar que essa característica do receituário impresso é vista como um avanço significativo e que tem contribuído para a legibilidade das prescrições. Sabe-se que uma fácil identificação e a escrita legível diminuem a possibilidade de erro na leitura e na dispensação feita pelo farmacêutico, e, consequentemente, ajudam o paciente na realização completa do tratamento. 0 processo de prescrição é complexo e permeado por erros. 0s erros são geralmente multifatoriais e originários de falhas ativas ou condições que induzem ao erro. Avaliar o perfil de legibilidade das receitas poderá ser útil na melhoria do processo de dispensação de medicamentos ${ }^{10}$.
Ao analisar as prescrições, sendo o maior número prescrito por clínico geral, pode-se associar esse fato, pois a Clínica Geral é a especialidade médica que mais possui atuação nas Unidades Básicas de Saúde ${ }^{11}$. Em outro estudo, realizado na Unidade Básica de Saúde da Vila Romana, em São Paulo, avaliando prescrições de pacientes que utilizavam medicamentos para hipertensão e diabetes, observou-se que os atendimentos dos pacientes nos níveis mais complexos, por especialidades médicas como cardiologia e endocrinologia, apresentaram um número limitado ou ausente ${ }^{12}$.

Em relação à origem do atendimento, observouse que a maior parte da população do município de Catunda recebe os medicamentos por meio do sistema público. Em um estudo realizado no Espírito Santo, avaliando a origem dos atendimentos de pacientes, analisou-se que apenas $15,8 \%$ da população é beneficiária de planos privados de saúde, sendo a maior parte da população dependente exclusivamente dos serviços ofertados pelo SUS. Sendo assim, o sistema público é a principal alternativa para viabilizar o tratamento medicamentoso, além de considerar a universalização da saúde pública através do SUS, sendo relevante relacionar esse fato com o perfil econômico do município ${ }^{13}$.

Os medicamentos mais usados para HAS foram 
Hidroclorotiazida, Losartana e Enalapril, porém, não apresentam diferenças significativas entre os diversos grupos farmacológicos no tratamento da hipertensão na prevenção de eventos cardiovasculares ${ }^{14}$. Enquanto que para DM, os medicamentos mais utilizados foram Metformina e Glibenclamida, sendo caracterizados como os medicamentos de primeira e segunda escolha, respectivamente. Identificou-se que os medicamentos mais utilizados pelos pacientes com DM, em um centro especializado no tratamento do diabetes, na Alemanha, foram os medicamentos para o trato alimentar e metabolismo, seguidos pelos utilizados no sistema cardiovascular. Em geral, a semelhança entre os medicamentos utilizados se deve às comorbidades mais prevalentes associadas ao DM, que, na maioria dos casos, é a hipertensão ${ }^{15}$.

Em um estudo realizado com participantes do grupo HIPERDIA, de Parobé, no Rio Grande do Sul, encontraram-se possiveis interações nas associações dos medicamentos, como: Glibenclamida + Hidroclorotiazida, Propanolol + Metformina, Enalapril + Metformina e Hidroclorotiazida + Metformina, sendo consideradas de grau leve a moderada ${ }^{10}$. Em outro estudo realizado na cidade de Parobé, citam-se interações frequentes como Enalapril + Hidroclorotiazida, seguidas de Enalapril + Glibenclamida. Por fim, a maioria dos pacientes utilizam mais de dois medicamentos, e, em relação às interações medicamentosas mais frequentes, pode-se realizar a educação em saúde com esses pacientes; desse modo, a educação em saúde pode ocorrer por meio do conhecimento sobre as possiveis interações medicamentosas e o desenvolvimento de ações bem planejadas ${ }^{16}$.

Os dados utilizados para compor esta pesquisa são os mesmos disponíveis para a consulta e planejamento de ações por parte das instituições de saúde responsáveis por acompanhar e estabelecer políticas públicas para essas comorbidades, assim, este estudo pode contribuir com o aprimoramento da análise dessas condições clínicas crônicas na população.

\section{CONCLUSÃO}

Este estudo apresentou resultados consideráveis referentes aos medicamentos de uso associado para hipertensão e diabetes presentes nas prescrições retidas na Unidade Básica de Saúde. Quanto ao sexo, as mulheres foram as que mais consumiram os medicamentos para hipertensão e diabetes. As prescrições apresentaram legibilidade, sendo a maioria preenchida de forma completa; a especialidade médica que mais prescreve é a Clínica Geral e com a origem de atendimento sendo majoritariamente na própria UBS. A Hidroclorotiazida (Diuréticos tiazídicos) foi o anti-hipertensivo mais prescrito, enquanto o hipoglicemiante mais prescrito foi a Metformina (Biguanidas). Quanto às possíveis interações medicamentosas, as mais frequentes foram Enalapril + Glibenclamida, seguida de Hidroclorotiazida + Glibenclamida e Propanolol + Metformina. Esses achados constituem informações importantes que podem contribuir no planejamento e execução de políticas públicas de saúde, assim como para a redução de outras complicações associadas, como doenças cardiovasculares.

Os dados obtidos neste estudo mostram que se fazem necessárias ações de educação em saúde para melhorar ainda mais o controle dessas patologias no município de Catunda, Ceará, com o intuito de garantir a qualidade de serviço prestado na saúde do município e, consequentemente, a qualidade de vida dos pacientes que fazem o uso dos medicamentos.

Portanto, os dados deste estudo podem instituir informações para o planejamento de ações tanto por parte do sistema público como pelos profissionais da saúde para acompanhar os pacientes com HAS e DM e, desse modo, evitar futuras complicações e gastos maiores de recursos públicos voltados a setor saúde.

\section{CONTRIBUIÇÃO DAS AUTORAS}

Maria Morgana Bezerra Costa contribuiu com a realização da pesquisa, o delineamento e a redação do manuscrito. Maria Isabel Linhares contribuiu com a revisão crítica no manuscrito.

\section{REFERÊNCIAS}

1. Malta DC, Bernal RTI, Oliveira M. Tendências dos fatores de risco de doenças crônicas não transmissiveis, segundo a posse de planos de saúde, Brasil, 2008 a 2013. Ciênc saúde colet. 2015; $20(40): 1005-16$.

2. Francisco PMSB, Segri NJ, Borim FSA, Malta $D C$. Prevalência simultânea de hipertensão e diabetes em idosos brasileiros: desigualdades individuais e contextuais. Ciênc saúde colet. 2018; 23(11):3829-40. doi: https://doi.org/10.1590/1413$\underline{812320182311.29662016}$ 
3. Malachias MVB, Plavnik FL, Machado CA, Malta D, Scala LCN, Fuchs S. $7^{\circ}$ Diretriz brasileira de hipertensão arterial. Braz J Hypertension. 2017; 24(1). doi: https://doi.org/ $10.5935 / a b c .20160151$

4. Riddle MC. Standards of Medical Care in Diabetes. J appl res educ. 2019; 42(1).

5. Freitas OS, Matta SR, Mendes LVP, Luiza VL, Campos MR. Uso de serviços de saúde e de medicamentos por portadores de Hipertensão e Diabetes no Município do Rio de Janeiro, Brasil. Ciênc saúde colet. 2018; 23(23):2383-92. doi: https:// doi.org/10.1590/1413-81232018237.21602016

6. Oliveira DM, Schoeller SD, Hammerschmidt KSA, Vargas MAO, Girondi JBR. Conhecimento da equipe de enfermagem nas complicações do diabetes mellitus em emergência. Acta Paul Enferm. 2014; 27(6):520-25. doi: https://doi.org/10.1590/1982$\underline{0194201400085}$

7. Conselho Nacional de Saúde. Resolução n. ${ }^{\circ}$ 466 de 12 de dezembro de 2012 [document on the internet]. 2012 [cited 2020 Out 18]. Available from: https://conselho.saude .gov.br/resolucoes/2012/Res0466.pdf

8. Amaral DMD, Perassolo MS. Possíveis interações medicamentosas entre os anti-hipertensivos e antidiabéticos em participantes do Grupo HIPERDIA de Parobé, RS (Uma análise teórica). Rev Cienc Farm Basica e Apl. 2012; 33(1):99-105. doi: https://doi. org/10.1590/1982-0194201400085

9. Mengue SS, Bertoldi AD, Ramos LR, Farias MR, Oliveira MA, Tavares NUL. Acesso e uso de medicamentos para hipertensão arterial no Brasil. Rev Saúde Pública. 2016; 50(2):1s-9s. https://doi.org/10.1590/S1518-8787.2016050006154

10. Araújo PTB, Uchôa SAC. Avaliação da qualidade da prescrição de medicamentos de um hospital de ensino. Ciênc saúde colet. 2011; 16:1107-14.

11. Melo D0, Silva SRA, Castro LLC. Avaliação de indicadores de qualidade de prescrição de medicamentos em uma unidade de atenção primária com diferentes modelos de atenção. Epidemiol Serv Saúde. 2016; 25:259-70.

12. Silva TR, Feldmam C, Lima MHA, Nobre MRC, Domingues RZL. Controle de diabetes Mellitus e hipertensão arterial com grupos de intervenção educacional e terapêutica em seguimento ambulatorial de uma Unidade Básica de Saúde. Saúde Soc. $2006 ; 15: 180-89$. doi: https://doi.org/10.1590/ $\underline{\text { S0104-12902006000300015 }}$
13. Boing $A C$, Bertoldi $A D$, Boing AF, Bastos JL, Peres KG. Acesso a medicamentos no setor público: análise de usuários do Sistema Único de Saúde no Brasil. Cad Saúde Pública. 2013; 29:691-701. doi: https://doi. org/10.1590/S0102-311X2013000400007

14. Turnbull F, Neal B, Ninomiya T, Algert C, Arima H, Barzi F. Effects of different regimens to lower blood pressure on major cardiovascular events in older and younger adults: metaanalysis of randomised trials. Bmj, 2008; 336(7653):1121-23. doi: https://doi.org/ 10.1136/bmj.39548.738368.BE

15. Silva MRR, Diniz LM, Santos JBR, Reis EA, Mata AR, Araújo VE. Uso de medicamentos e fatores associados à polifarmácia em indivíduos com diabetes mellitus em Minas Gerais, Brasil. Ciênc saúde colet. 2018; 23:2565-74. doi: https://doi.org/10.1590/1413$\underline{81232018238.10222016}$

16. Rempel C, Goettert MI, Strohschoen AAG, Carreno I, Manfroi M, Moreschi C. Análise da medicação utilizada por diabéticos e hipertensos. Rev Caderno Pedagógico [serial on the internet]. 2015 [cited 2020 Nov 28]; 12(1)242-52. Available from: http://www. univates.br/revistas/index.php/cadped/article/ view $/ 948 / 936$
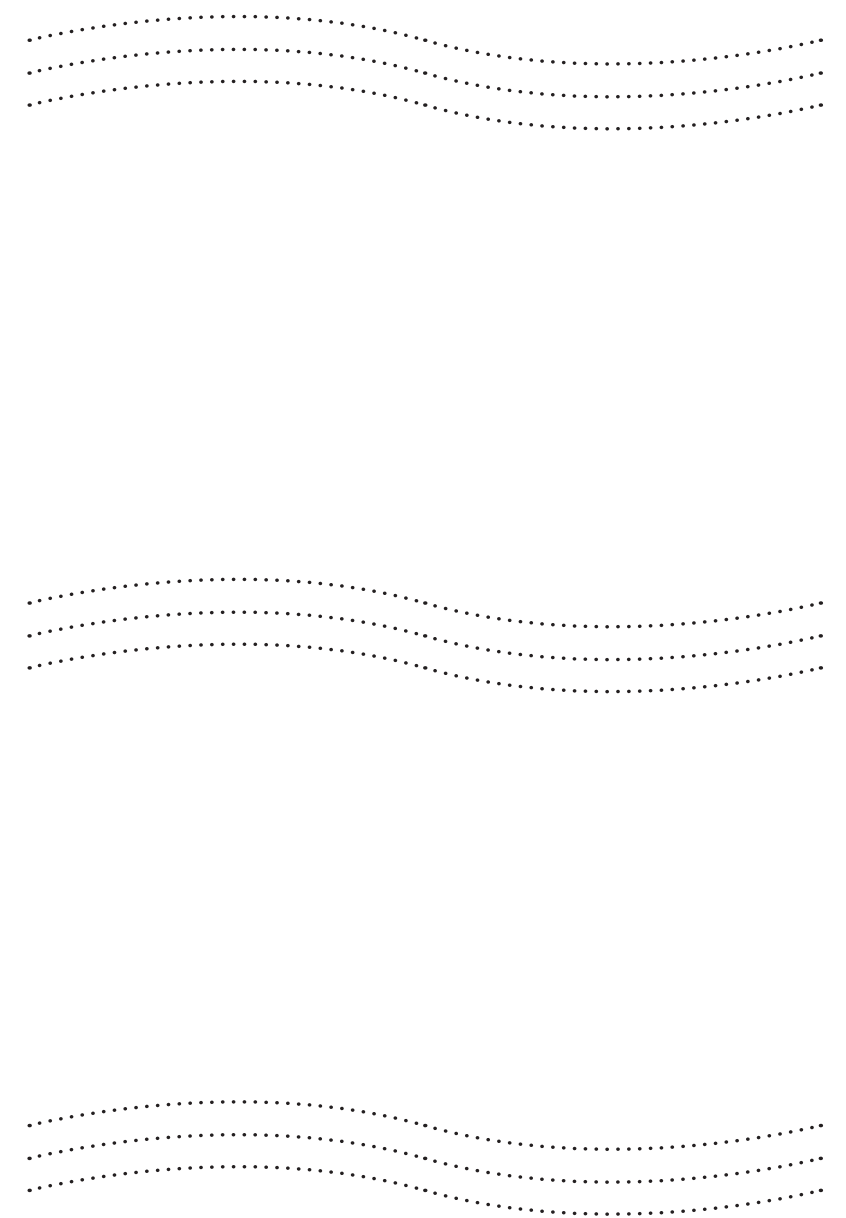